\title{
Yellow nail syndrome after allogeneic haematopoietic stem cell transplantation in two patients with multiple myeloma
}

\section{Céline Gregoire, Julien Guiot, Gaëlle Vertenoeil, Évelyne Willems, Kaoutar Hafraoui, Jean-Louis Corhay, Renaud Louis \& Yves Beguin}

To cite this article: Céline Gregoire, Julien Guiot, Gaëlle Vertenoeil, Évelyne Willems, Kaoutar Hafraoui, Jean-Louis Corhay, Renaud Louis \& Yves Beguin (2016) Yellow nail syndrome after allogeneic haematopoietic stem cell transplantation in two patients with multiple myeloma, Acta Clinica Belgica, 71:6, 428-430, DOI: 10.1080/17843286.2015.1122872

To link to this article: http://dx.doi.org/10.1080/17843286.2015.1122872

曲 Published online: 06 Feb 2016.

Submit your article to this journal $\sqsubset$

Wll Article views: 37

Q View related articles $\asymp$

View Crossmark data ¿

Citing articles: 1 View citing articles $\square$ 


\title{
Yellow nail syndrome after allogeneic haematopoietic stem cell transplantation in two patients with multiple myeloma
}

\author{
Céline Gregoire $^{1}$, Julien Guiot ${ }^{2}$, Gaëlle Vertenoeil ${ }^{1}$, Évelyne Willems ${ }^{1}$, Kaoutar \\ Hafraoui', Jean-Louis Corhay², Renaud Louis' ${ }^{2}$, Yves Beguin ${ }^{1}$
}

${ }^{1}$ Department of Medicine, Division of Hematology, CHU of Liège and University of Liège, Liège, Belgium, ${ }^{2}$ Department of Medicine, Division of Pneumology, CHU of Liège and University of Liège, Liège, Belgium

\begin{abstract}
Objective and Importance: Yellow nail syndrome (YNS) is a rare disorder of unknown aetiology characterized by the triad of yellow nails, lymphoedema and respiratory manifestations. About 200 cases have been reported, but a lot of patients probably elude proper diagnosis because of both variability of symptoms and ignorance of this syndrome by many physicians. The pathogenesis remains unclear, and could involve functional lymphatic abnormalities, microvasculopathy or lymphocyte deficiency, but none of these hypotheses seems fully satisfactory.

Clinical Presentation: We report for the first time two cases of YNS associated with multiple myeloma relapsing after non-myeloablative haematopoietic cell transplantation (HCT). In these two cases, onset or worsening of YNS symptoms followed graft-versus-host disease (GvHD) manifestations.
\end{abstract}

Intervention: Corticosteroids given to treat GvHD also improved YNS manifestations.

Conclusion: YNS after HCT might be a microvascular manifestation of endothelial GvHD and corticosteroids might be an effective treatment.

Keywords: Yellow nail syndrome, Allogeneic haematopoietic cell transplantation, Graft-versus-host-disease, Multiple myeloma

\section{Introduction}

Yellow nail syndrome (YNS) is a rare disorder of unknown aetiology characterized by the triad of yellow nails, lymphoedema and respiratory manifestations. It was first described by Samman and White in 1964 and about 200 cases have been reported. We report for the first time two cases of YNS associated with graft-versus-host-disease (GvHD) after non-myeloablative (NMA) haematopoietic cell transplantation (HCT) for multiple myeloma (MM).

\section{Case 1}

The first patient was diagnosed with light chain MM at the age of 41 and treated by chemotherapy, three autologous HCT and an allogeneic NMA-HCT, achieving a very good partial response (VGPR). Immunosuppressive regimen was based on the association of mycophenolate mofetil (MMF) and tacrolimus, discontinued at day 42 and day 240 post-transplantation, respectively. Fifteen

Correspondence to: Céline Gregoire, Department of Hematology, University of Liège, CHU Sart Tilman, 4000 Liège, Belgium. Email: celine. gregoire@ulg.ac.be months after transplantation, the patient developed chronic cutaneous GvHD, respecting the nails, concurrently with bilateral lower limb oedema, sinusitis and pleuro-pericardial effusion. The pleural fluid was a monocytic exudate (white blood cells $690 / \mu \mathrm{L}$ with $21 \%$ lymphocytes and $78 \%$ monocytes, proteins $39 \mathrm{~g} / \mathrm{L}$, lactate dehydrogenase 202 UI/L), without any micro-organism or malignant cell. These manifestations were identified as GvHD-associated serositis and partially improved with corticosteroids, ciclosporine and furosemide. Except for limited probable lung aspergillosis (treated initially with voriconazole, then with amphotericin B), the patient remained stable for six months, until a surgical procedure was complicated by respiratory distress. Thoracic scanner showed pleuro-pericardial effusion, ground grass infiltrates and nodular lesions. Antibiotic, antifungal and diuretic therapy led to a favourable evolution. Three months later, MM relapsed and was treated successively with thalidomide and bortezomib (allowing a VGPR), while immunosuppressive drugs were stopped. Several months later, the patient developed a bronchopneumonia with pleuro-pericardial 


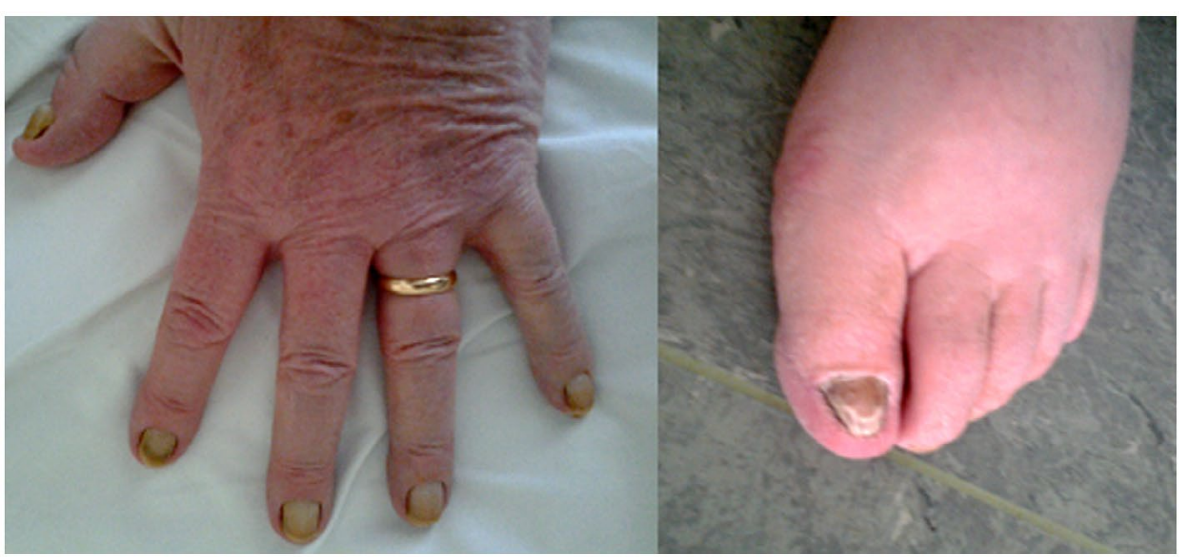

Figure 1 Yellow nails. Ungueal dystrophy (patient 2): nails are yellow, thickened, with excessive curvature and onycholysis.

effusion, concurrently with a new myeloma progression. After failure of bortezomib and dexamethasone, the former was replaced by lenalidomide, and complete remission (CR) as well as significant improvement of the cardiopulmonary manifestations were observed. Chronic symptoms of sinusitis, respiratory infections and pleuro-pericardial effusion fluctuated during two years, but significantly worsened, together with apparition of onycholysis, when ocular GvHD started. After an initial improvement of anasarca with furosemide, the patient developed a septic shock of pulmonary origin, with pleuritis and pericardial lymphocytic effusion, requiring a pleura-pericardial window. YNS was then diagnosed, as yellow nails were observed, together with bronchopneumonia, lower limb lymphedema and bilateral pleural effusion. After a good response to empirical antibiotherapy, long-term treatment consisted in pulmonary rehabilitation, low-dose long-term corticosteroid therapy and topical vitamin E applied to the nails. The evolution has been excellent, with less invalidating ocular symptoms, less frequent respiratory infections and sinusitis, and reduced dyspnoea and lymphoedema, but no objective improvement of pulmonary function (restrictive and small airways obstructive syndrome) and nail abnormalities, and persisting ground glass opacities on CT-scan.

\section{Case 2}

The second patient was diagnosed with immunoglobulin G kappa MM at the age of 62, and treated with chemotherapy and double autotransplantation, followed by a NMA-HCT from a HLA-identical sibling, leading to a CR. Immunosuppressive regimen was based on the association of MMF, discontinued at day 28 post-transplantation, and tacrolimus. While still receiving tacrolimus, the patient developed cutaneous and digestive acute GvHD, treated with corticosteroids. Two months later, while corticosteroids were being tapered, he developed an autoimmune haemolytic anaemia secondary to Epstein-Barr virus reactivation (resistant to corticosteroids, intravenous immunoglobulins and rituximab, eventually cured after splenectomy). Around 15 months later, as all immunosuppressive treatments had long been stopped, he suffered from tetraparesis and tabetic ataxia caused by a demyelinating inflammatory myelitis, whose aetiology was probably dysimmune (infectious and inflammatory causes having been excluded). This myelitis was successfully treated with corticosteroids, which were tapered over 2 months. In this context of immunosuppression and hypogammaglobulinemia, the patient had recurrent respiratory tract infections and sinusitis. One month after discontinuation of corticosteroids, ungueal dystrophy was noted for the first time (Fig. 1), without any other sign of GvHD. Approximately one year later, myeloma relapsed, and donor lymphocytes infusion (DLI) allowed a new CR. Eighteen months after DLI, the patient suffered from ocular GvHD, treated with topical corticosteroids. One month later, he developed abundant right pleural effusion and non-pitting lower limb edema, associated with diffuse broncho-bronchiolopathic syndrome, mediastinal lymphadenopathies and a moderate restrictive syndrome with diminished diffusing capacity for carbon monoxide. Renal and cardiac functions were normal. The pleural fluid was a haemorrhagic lymphocytic exudate (red blood cells $11,600 / \mu \mathrm{L}$, white blood cells $6264 / \mu \mathrm{L}$ with $74 \%$ lymphocytes and $24 \%$ monocytes, lactate dehydrogenase $100 \mathrm{UI} / \mathrm{L}$, proteins $52 \mathrm{~g} / \mathrm{L}$ ), without micro-organism or malignant cell. Diuretics allowed an initial resolution of symptoms, but they reappeared, associated with yellow dystrophic nails, leading to the diagnosis of YNS. The diuretic treatment was intensified, but symptoms recurred after two bronchopneumonias with pleural effusion requiring iterative drainage and eventually a pleuroscopy with talc pleurodesis. Biopsies of the pleura showed chronic lymphocytic pleuritis. As ocular GvHD worsened, the patient received a three-week course of systemic corticosteroids, allowing a healing of the keratitis, as well as a partial regression of symptoms of YNS, with less yellow nails, diminished lymphoedema and less frequent sinusitis. 


\section{Discussion}

YNS is characterized by the triad of yellow nails, lymphoedema and respiratory manifestations. As the manifestations are inconstant, only two of the three features are required. A lot of patients probably elude proper diagnosis because of both variability of symptoms and ignorance of this syndrome by many physicians. Nails may be yellow with slow growth, thickening, excessive curvature or onycholysis. Non-pitting bilateral lymphedema of lower extremities is often present. ${ }^{1}$ Cardio-respiratory manifestations include cough, dyspnoea, pericardial and bilateral pleural effusions, bronchiectasis, respiratory tract infections and chronic sinusitis. Pleural fluid is typically an exudate by the protein criterion but a transudate by the lactate dehydrogenase criterion, sometimes a chylothorax. Cellular analysis shows lymphocytic predominance and no micro-organism. Pulmonary function is frequently abnormal, with a reversible obstructive pattern most commonly observed; restrictive lung defects are less common and usually associated with the presence of a pleural effusion (as seen in our two patients). ${ }^{2}$ Treatment of YNS is essentially symptomatic. Topical steroids or vitamin E are sometimes applied to the nails. Improvements of nail abnormalities and lymphoedema have been noted with better control of respiratory manifestations. ${ }^{1}$ In patients with immune deficiency, immunoglobulin administration may be useful. ${ }^{3}$ In our patients, corticotherapy allowed an improvement of both GvHD and YNS manifestations.

YNS has been described in association with a variety of conditions ${ }^{1,3}$ such as malignancies, including lung cancer ( 4 cases), breast cancer ( 4 cases), or haematologic malignancies (1 case of macroglobulinemia, 2 cases of non-Hodgkin's lymphoma and 1 case of mycosis fungoides), immunodeficiencies (12 cases), rheumatoid arthritis (7 cases), thiol compounds such as bucillamine used in rheumatoid arthritis (41 cases), titanium released by implants (34 cases), tuberculosis (3 cases), nephrotic syndrome ( 2 cases), exudative enteropathy ( 2 cases $), \ldots$ YNS has never been described as an association with MM or GvHD. In our cases, some of the manifestations appeared a few months before diagnosis of disease relapse, and were improved after treatment of these relapses. Nevertheless, this association was inconstant as patients in CR of their MM were still suffering from YNS symptoms. It rather appears that onset or worsening of YNS symptoms followed GvHD manifestations.
The pathogenesis of YNS remains unclear. A first series of hypotheses involved functional or anatomic lymphatic abnormalities. Nail manifestations might be due to ectasia of endothelium-lined lymphatic vessels or to lipofuscin, ${ }^{1}$ and recurrent airway infections might be due to impaired lymphatic drainage, resulting in delayed bacterial clearance. Conversely, infections are considered by some as a trigger overwhelming a 'saturated' dysfunctional lymphatic network. ${ }^{1}$ Some authors have also suggested that YNS might be due to lymphocyte deficiency, secondary to their migration into pleural fluid and dermis. ${ }^{3} \mathrm{~A}$ more recent hypothesis involves microvasculopathy with increased capillary permeability and protein leakage, resulting in pleural effusion (with a high protein content), as well as hypogammaglobulinemia and lymphopenia contributing to the high rate of respiratory infections. ${ }^{4}$ This theory is supported by the reports associating YNS and exsudative enteropathy or nephrotic syndrome. There is also increasing evidence of the involvement of endothelial damage by alloreactive $\mathrm{T}$ cells in GvHD, in addition to the classic epithelial damage..$^{5-7}$ In our cases, YNS might therefore be a microvascular manifestation of GvHD.

\section{Conclusion}

YNS is a disorder characterized by yellow nails, lymphoedema and respiratory manifestations. We report the first two cases of YNS associated with MM and NMA-HCT. In these cases, YNS might be a microvascular manifestation of GvHD and corticosteroids might be an effective treatment.

\section{References}

1 Maldonado F, Ryu JH. Yellow nail syndrome [Review]. Curr Opin Pulm Med. 2009;15:371-5.

2 Maldonado F, Tazelaar HD, Wang CW, Ryu JH. Yellow nail syndrome: analysis of 41 consecutive patients. Chest. 2008;134:375-81.

3 Gupta S, Samra D, Yel L, Agrawal S. T and B cell deficiency associated with yellow nail syndrome. Scand J Immunol. 2011;75:29-335.

4 D’Alessandro A, Muzi G, Monaco A, Filiberto S, Barboni A, Abbritti G. Yellow nail syndrome: does protein leakage play a role? Eur Respir J. 2001;17:149-52.

5 Gyger M, Rosenberg A, Shamy A, Hercz A, Caplan S, Sebag IA, et al. Vascular leak syndrome and serositis as an unusual manifestation of chronic graft-versus-host disease in nonmyeloablative transplants. Bone Marrow Transplant. 2005;35:201-203.

6 Tichelli A, Gratwohl A. Vascular endothelium as 'novel' target of graft-versus-host disease [Review]. Best Pract Res Clin Haematol. 2008;21:139-48.

7 Penack O, Socie G, van den Brink MR. The importance of neovascularization and its inhibition for allogeneic hematopoietic stem cell transplantation. Blood. 2011;117:4181-9. 